\title{
BMJ Open Comprehensive public health evaluation of lockdown as a non- pharmaceutical intervention on COVID-19 spread in India: national trends masking state-level variations
}

\author{
Maxwell Salvatore, ${ }^{1,2}$ Deepankar Basu, ${ }^{3}$ Debashree Ray, ${ }^{4,5}$ Mike Kleinsasser, \\ Soumik Purkayastha (1) , ${ }^{1}$ Rupam Bhattacharyya, ${ }^{1}$ Bhramar Mukherjee (1) ${ }^{1,2}$
}

To cite: Salvatore M, Basu D, Ray D, et al. Comprehensive public health evaluation of lockdown as a nonpharmaceutical intervention on COVID-19 spread in India: national trends masking statelevel variations. BMJ Open 2020;10:e041778. doi:10.1136/ bmjopen-2020-041778

- Prepublication history and additional material for this paper is available online. To view these files, please visit the journal online (http://dx.doi.org/10. 1136/bmjopen-2020-041778)

Received 17 June 2020

Revised 02 November 2020

Accepted 11 November 2020

Check for updates

(C) Author(s) (or their employer(s)) 2020. Re-use permitted under CC BY-NC. No commercial re-use. See rights and permissions. Published by BMJ.

For numbered affiliations see end of article.

Correspondence to

Professor Bhramar Mukherjee; bhramar@umich.edu

\section{ABSTRACT}

Objectives To evaluate the effect of four-phase national lockdown from March 25 to May 31 in response to the COVID-19 pandemic in India and unmask the state-wise variations in terms of multiple public health metrics.

Design Cohort study (daily time series of case counts). Setting Observational and population based.

Participants Confirmed COVID-19 cases nationally and across 20 states that accounted for $>99 \%$ of the current cumulative case counts in India until 31 May 2020.

Exposure Lockdown (non-medical intervention).

Main outcomes and measures We illustrate the masking of state-level trends and highlight the variations across states by presenting evaluative evidence on some aspects of the COVID-19 outbreak: case fatality rates, doubling times of cases, effective reproduction numbers and the scale of testing.

Results The estimated effective reproduction number R for India was 3.36 (95\% Cl 3.03 to 3.71$)$ on 24 March, whereas the average of estimates from 25 May to 31 May stands at 1.27 (95\% Cl 1.26 to 1.28). Similarly, the estimated doubling time across India was at 3.56 days on 24 March, and the past 7-day average for the same on 31 May is 14.37 days. The average daily number of tests increased from 1717 (19-25 March) to 113372 (25-31 May) while the test positivity rate increased from $2.1 \%$ to $4.2 \%$, respectively. However, various states exhibit substantial departures from these national patterns. Conclusions Patterns of change over lockdown periods indicate the lockdown has been partly effective in slowing the spread of the virus nationally. However, there exist large state-level variations and identifying these variations can help in both understanding the dynamics of the pandemic and formulating effective public health interventions. Our framework offers a holistic assessment of the pandemic across Indian states and union territories along with a set of interactive visualisation tools that are daily updated at covind19.org.

\section{INTRODUCTION}

COVID-19 is an infectious disease caused by SARS-CoV-2. ${ }^{1}$ First identified in December 2019 in Wuhan, China, it has since spread

\section{Strengths and limitations of this study}

- Provides one of the first comprehensive nationwide as well as state-level evaluation of the effect of national lockdown on progression of COVID-19 in India using an ensemble of public health metrics.

- Provides a set of public health metrics displayed via interactive visualisation tools that are daily updated at covind19.org to help inform dynamic policy towards containment and mitigation.

- Introduces the concept of a cascade of 'peaks' across states instead of a solitary and unique national peak for the daily virus incidence curve.

- Metrics presented in this study do not include predictions of future daily active cases nor account for age-sex structure and mobility patterns and thus do not inform us about projected healthcare needs.

- Does not assess the degree of under-reporting in cases and deaths and does not consider broader and long-term impacts of the lockdown, for example, the economic and social costs.

globally, resulting in an ongoing pandemic. ${ }^{2}$ As of 9 June 2020, at the time of writing this paper, more than 7 million cases have been reported across 188 countries and territories, resulting in more than 405000 deaths. India, a democracy of 1.35 billion with a high population density and fragile healthcare infrastructure, is one of the global epicentres for this pandemic. The first reported coronavirus infection in India was on 30 January 2020 and was identified as being imported by travel. The government of India had initially responded to the pandemic with closing its borders and suspending all visas. With the pandemic accelerating throughout the world, the government began issuing advisories regarding social distancing measures and eventually, India implemented a strict nationwide lockdown from 25 March until 
31 May $2020,{ }^{3}$ after which phased lockdown for containment zones is in effect until 30 June $2020 .{ }^{4}$ The government implemented a zonal classification of regions in the nation, with each region falling in one of three classesred zones (hotspots with high doubling rates and high number of active cases), orange zones (non-hotspots with fewer cases) and green zones (regions without confirmed cases or without new cases in the previous 21 days). In addition to nationwide response patterns, Indian state governments responded to the pandemic with various declarations of emergency, closure of institutions and public meeting places, in addition to other restrictions to contain the spread of the virus. Table 1 provides an overview of said variations over the four phases of lockdown in India. As of 11 June, the number of total confirmed cases in India has crossed 298 000, of whom 8501 have died and 146972 have recovered, placing India at a worldwide rank of 4 in terms of total confirmed cases. ${ }^{5}$ The number of new

Table 1 National and state-level lockdown measures implemented over the course of COVID-19 pandemic in India

\begin{tabular}{|c|c|c|}
\hline Lockdown phase & Nationwide measures implemented & State-level variation in measures implemented \\
\hline $\begin{array}{l}\text { Phase } 1 \\
\text { (25 March to } 14 \text { April) }\end{array}$ & $\begin{array}{l}\text { All transport services-road, air and rail-were } \\
\text { suspended, with exceptions for transportation } \\
\text { of essential goods, fire, police and emergency } \\
\text { services. Educational institutions, industrial } \\
\text { establishments and hospitality services were also } \\
\text { suspended.* Services such as food shops, banks } \\
\text { and ATMs, petrol pumps, other essentials and their } \\
\text { manufacturing were exempted. } \dagger\end{array}$ & $\begin{array}{l}\text { Gujarat, Himachal Pradesh, Karnataka, } \\
\text { Maharashtra, Tamil Nadu, Sikkim and Telangana } \\
\text { sealed state borders. Additionally, Maharashtra, } \\
\text { Telangana and Tamil Nadu imposed Section 144, } \\
\text { outlawing large gatherings of people.‡ }\end{array}$ \\
\hline
\end{tabular}

Phase 2

(15 April to 3 May)

\section{Conditional relaxation promised after 20 April,}

subject to containment of spread. Lockdown areas classified into red, orange and green zones based on extent of spread of disease. Certain relaxations from 20 April: agricultural businesses, including dairy, aquaculture and plantations allowed to open. Cargo transportation vehicles allowed to operate. Banks and government centres distributing benefits allowed to open as well.§

$\begin{array}{ll}\text { Phase } 3 & \text { Zonal classification of regions into red, orange and } \\ \text { (4-17 May) } & \text { green zones continued, with normal movement } \\ & \text { allowed in green zones. Movement of private and } \\ & \text { hired vehicles allowed in orange zones and red } \\ & \text { zones remained in lockdown. Zonal classifications } \\ & \text { revised on a weekly basis. }{ }^{* \star}\end{array}$

Phase 4 (18-31 May)
Unlike the previous phases, states were given a larger say in the demarcation of green, orange and red zones and the implementation roadmap. Red zones were further divided into containment and buffer zones. Local administrative bodies were given the authority to demarcate containment and buffer zones. 㧊
In interest of economic recovery, certain states like Maharashtra chose to allow specific business activities to resume, in addition to national easing of restrictions. Karnataka chose to ease the lockdown in certain areas, while Delhi, Punjab and Telangana chose to enforce strict lockdown measures. ๆ

Delhi allowed public and private-sector offices to reopen, with social distancing measures in place. Maharashtra eased most industrial and commercial activities. Gujarat and Jharkhand allowed no relaxation, while Bihar, Uttar Pradesh, Rajasthan and Madhya Pradesh chose to mostly adhere to guidelines issued by the Union Home Ministry. $\dagger^{\dagger}$ Restricted individual movement allowed in Delhi, while Maharashtra, Tamil Nadu and Telangana extended the lockdown further. Karnataka allowed public transport with social distancing measures, while West Bengal began easing workplace restrictions. Stand-alone shops were allowed to open for short durations.§§

\footnotetext{
*Guidelines on measures to be undertaken by ministries/departments of government of India, state/union territory governments and state/ union territory authorities for containment of COVID-19 epidemic in the country (https://www.mha.gov.in/sites/default/files/Guidelines.pdf). †The Economic Times: India's 21-day lockdown to counter coronavirus: what's exempt, what's not, 25 March 2020 (https://economictimes. indiatimes.com/news/politics-and-nation/india-21-day-lockdown-what-is-exempted-what-is-not/articleshow/74798725.cms). †Wikipedia: https://en.wikipedia.org/wiki/Indian_state_government_responses_to_the_COVID-19_pandemic. §BBC: Coronavirus lockdown guidelines: what has India changed under new rules? 15 April 2020 (https://www.bbc.com/news/world-asiaindia-52290761).

IHindustan Times: Complete list of states with no relaxation in lockdown 2.0 restrictions, 20 April 2020 (https://www.hindustantimes.com/ india-news/complete-list-of-states-with-no-covid-19-lockdown-2-0-relaxation/story-pfE5K3Pn5LSZrgFEvC84hO.html).

**India Today: Full list of red, yellow, green zone districts for lockdown 3.0, 1 May 2020 (https://www.indiatoday.in/india/story/red-orangegreen-zones-full-current-update-list-districts-states-india-coronavirus-1673358-2020-05-01).

††Hindustan Times: COVID-19 lockdown 3.0: a look at relaxations, restrictions across major states in India, 4 May 2020 (https://www. hindustantimes.com/india-news/coronavirus-update-covid-19-lockdown-3-0-a-look-at-relaxations-restrictions-across-major-states-in-india/ story-J5Z2lypwiagUTFf1wYW0jN.html).

抽e Economic Times: Lockdown 4.0 guidelines: nationwide lockdown extended until 31 May, with considerable relaxations, 21 May 2020 (https://economictimes.indiatimes.com/news/politics-and-nation/centre-extends-nationwide-lockdown-till-may-31-with-considerablerelaxations/articleshow/75790821.cms).

§BBC: India lockdown 4.0: what is allowed in your city? 19 May 2020 (https://www.bbc.com/news/world-asia-india-52707371).
} 


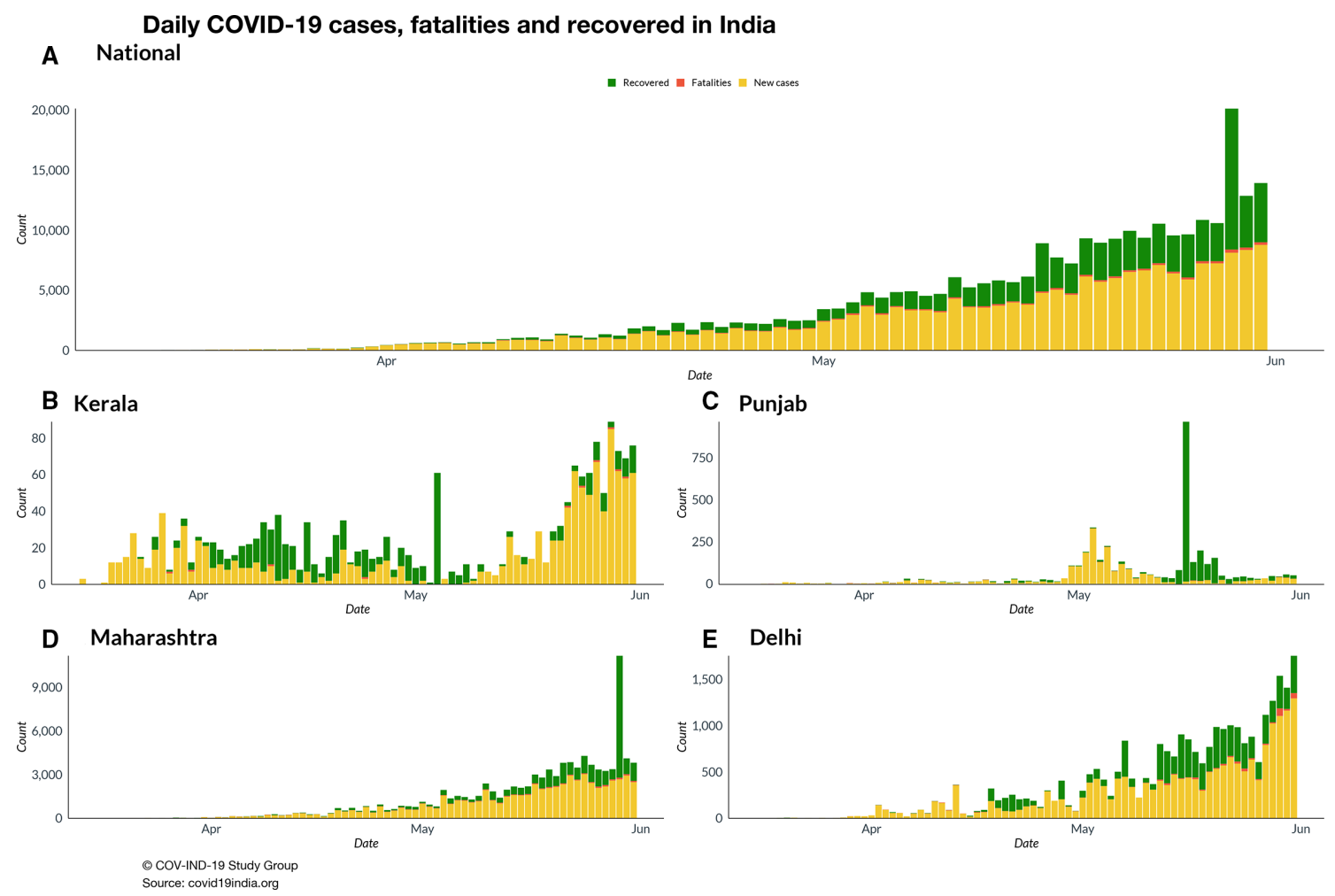

Daily COVID-19 cases, fatalities and recovered in India

Figure 1 Daily number of reported cases, fatalities and recovered cases in India (panel A) over the period between 15 March and 31 May with four states to capture the variation. Kerala (panel B) was doing well initially but has seen a recent surge of cases. Punjab (panel C) is an example state of 'doing well' whereas case counts in Maharashtra (panel D) and Delhi (panel E) are still increasing.

cases in India is not on the decline even after 9 weeks of national lockdown. There is state-level variability in terms of non-medical interventions and with respect to testing patterns (both in terms of testing strategies as well as test kits being used). The tests primarily used are the rapid antigen tests and reverse transcription PCR (RT-PCR) tests, with the former being given priority in containment zones and points of entry, while the latter are more widely used in non-containment areas and hospital settings. ${ }^{6}$

In light of tremendous public health interest, numerous data repositories, along with statistical models, are being developed with the aim of studying the effect of COVID-19 non-medical interventions. The focus of modelling is shifting from forecasting to evaluation of the effect of various interventions on the spread of the virus. ${ }^{7}$ As of 9 June, 4880 COVID-19 SARS-CoV-2 preprints have been uploaded to medRxiv and bioRxiv, of which at least 30 focus on analysing the efficacy of the non-medical interventions implemented by the Indian government. Ray and colleagues studied the short-term and long-term impacts of the initial lockdown on the total number of cases in India using standard epidemiological forecasting models, and concluded that the lockdown stood a good chance of reducing the total number of cases in India in the short term. ${ }^{8}$ Looking at several metrics, Mitra and colleagues suggested that curtailment strategies employed by the Indian government seem to have been effective in controlling the spread of the pandemic in the country. ${ }^{9}$ Ghosh and colleagues investigated the spread of the virus and subsequent impact of preventive measures on the same at a state level in India and noted that the lockdown has had differential effects on daily infection rates for various states in India. ${ }^{10}$ Jakhar and colleagues modelled data released by the Indian Ministry of Health and Family Welfare using the classical susceptible-infected-recovered (SIR) model and calculated the basic reproduction number $\left(R_{0}\right)$ for India as a whole, along with state-specific values of the same. ${ }^{11}$ Similarly, Gupta estimated key epidemiological parameters and evaluated the effect of control measures on the COVID-19 epidemic in India and its states using a dynamic compartment-based susceptibleexposed-infected-removed (SEIR) modelling approach, reiterating that state-specific $\mathbf{R}_{0}$ values exhibit high variability with respect to the national value of $\mathrm{R}_{0} \cdot{ }^{12}$ However, much is left to be done now that the nationwide lockdown has ended and a targeted lockdown phase is ongoing. All epidemiological projections suggest that current gains may be reversed rapidly if air travel and social mixing resume. For the time being, the general guideline is to reopen the country in a phased manner. ${ }^{13}$ The need of the hour is to study and analyse infection, recovery and fatality trends at a more granular level using multiple measures of assessing epidemic dynamics to ensure the formulation of targeted and customised interventions aimed at containment and mitigation.

In this paper, we consider an ensemble of metrics including case and death counts, case fatality rates (CFR), effective basic reproduction numbers, doubling times (DT) and assessment 

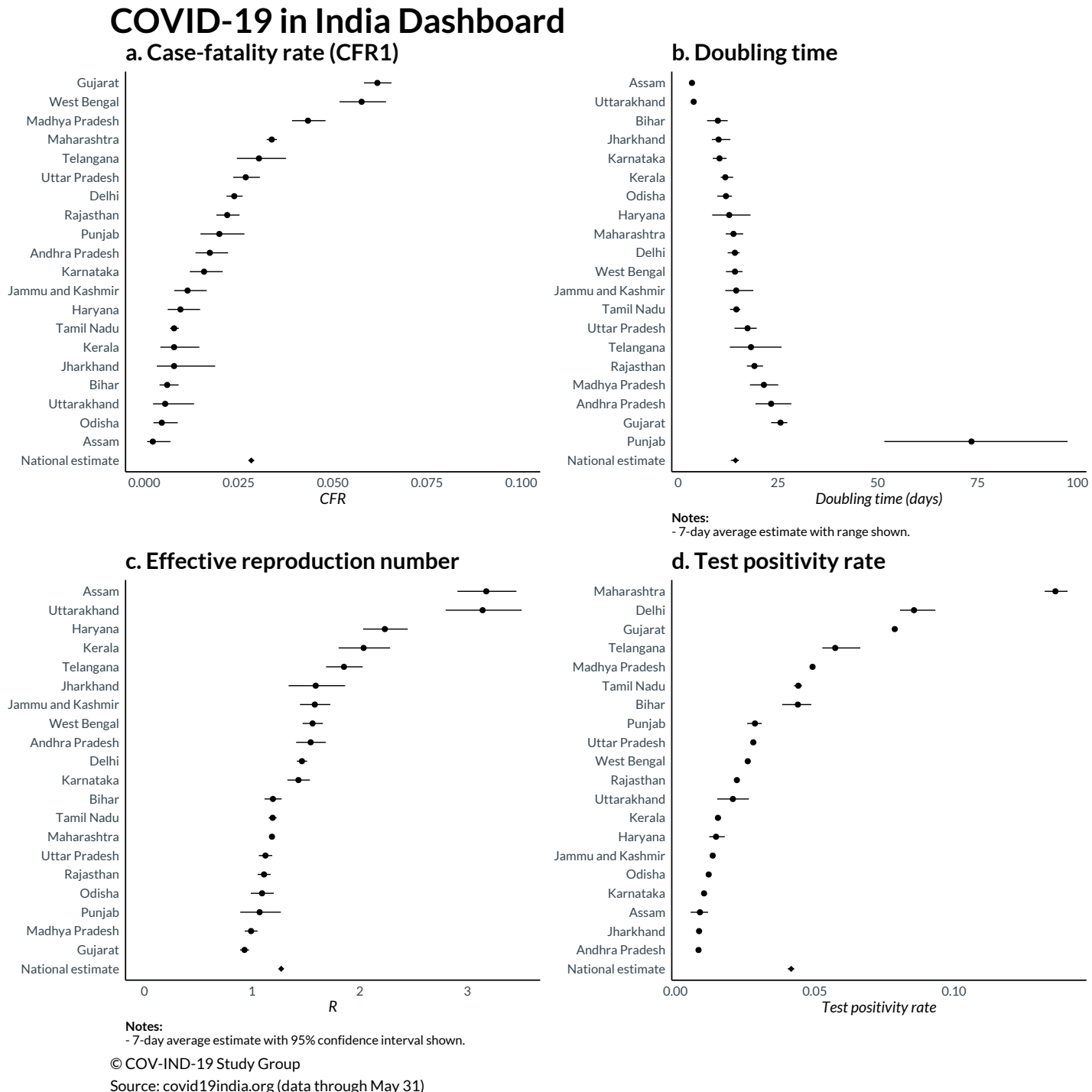

(c) COV-IND-19 Study Group

Source: covid19india.org (data through May 31)

Figure 2 Forest plot dashboard. (A) Forest plot of estimated case fatality rates (CFR1) based on all confirmed cases as of 31 May, along with $95 \% \mathrm{Cls}$, for 20 states and union territories of India, and a national summary.(B) Forest plot of estimated doubling times (in days) based on data from a 7-day past window from 31 May, along with 95\% Cls, for 20 states and union territories of India, and a national summary. (C) Forest plot of estimated time-varying R (effective basic reproduction number) based on data from a 7-day past window from 31 May, along with $95 \%$ Cls, for 20 states and union territories of India, and a national summary. (D) Forest plot of test positivity rates (proportion scale) based on data as of 31 May, for 20 states and union territories of India, along with a national summary.

of testing across states for a deeper and policy-relevant understanding of the COVID-19 situation in India after four contiguous periods of lockdown from 25 March to 31 May (lockdown 1.0: 25 March to 14 April, ${ }^{14}$ lockdown 2.0: 15 April to 3 May, ${ }^{15}$ lockdown 3.0: 4 May to 17 May, ${ }^{16}$ lockdown 4.0: 18 May to $31 \mathrm{May}^{17}$ ). By studying the series of natural experiments across the states and learning from their successes and failures, one has a better likelihood of designing improved targeted interventions for the next phase of the pandemic. Our proposed comprehensive dashboard has broader utility for policymakers and the supporting interactive platform presents daily updates for all metrics and models.

\section{METHODS}

We use publicly available data for all our analyses (covid19india.org and Our World In Data) ${ }^{5}{ }^{18}$ All source code and interactive plots are available at covind19.org. ${ }^{19}$ All computations were done using the RStudio platform.

\section{Case and death counts and fatality rates}

In addition to simple case and death counts, we look at CFRs estimated using all confirmed cases (CFR1, ratio of the total number of deaths and the total number of cases) and closed cases only (CFR2, ratio of the total number of deaths and the sum of the same and the total number of recovered cases). We construct appropriate CIs for these measures. ${ }^{20}$

DTs and growth rates/reproduction number

To quantify the growth of the pandemic, we estimated DTs for total confirmed cases using a 7-day backward-looking 


\section{A Doubling time}

trailing 7-day interval; data through May 31

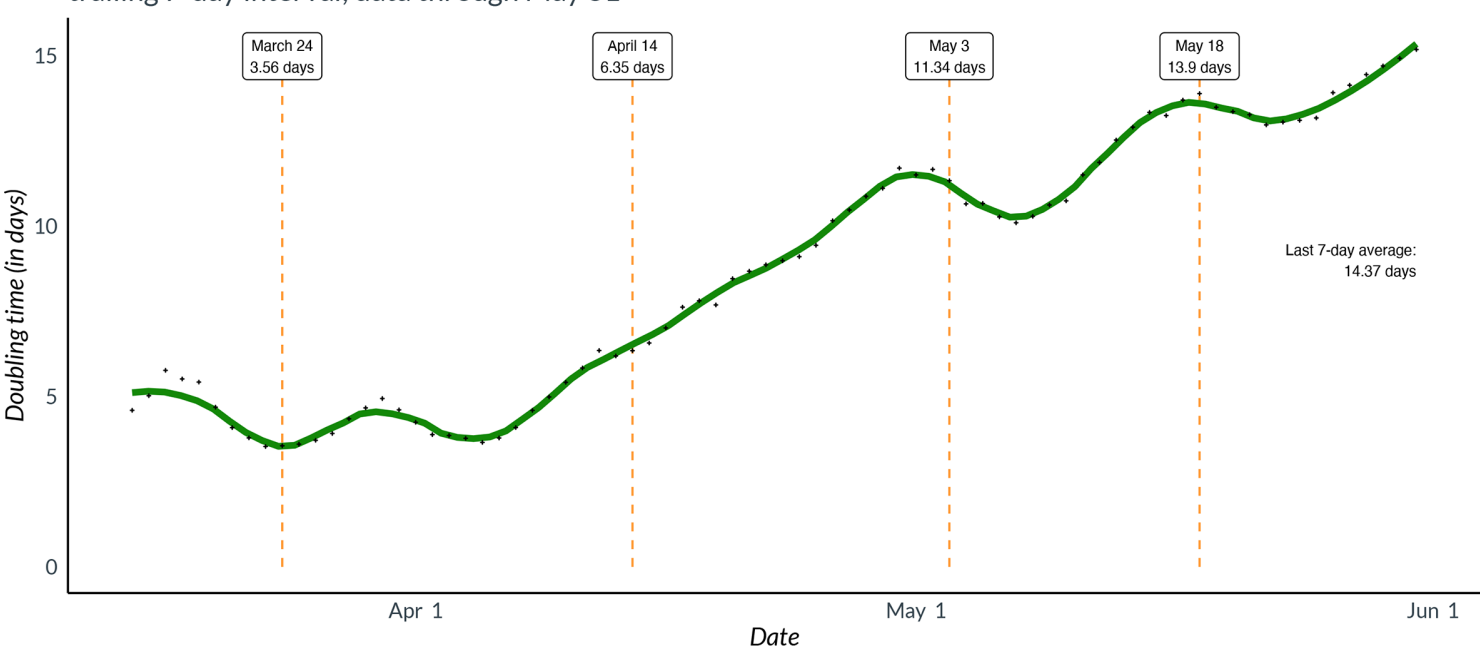

B Time-varying $\mathbf{R}$

as of May 31

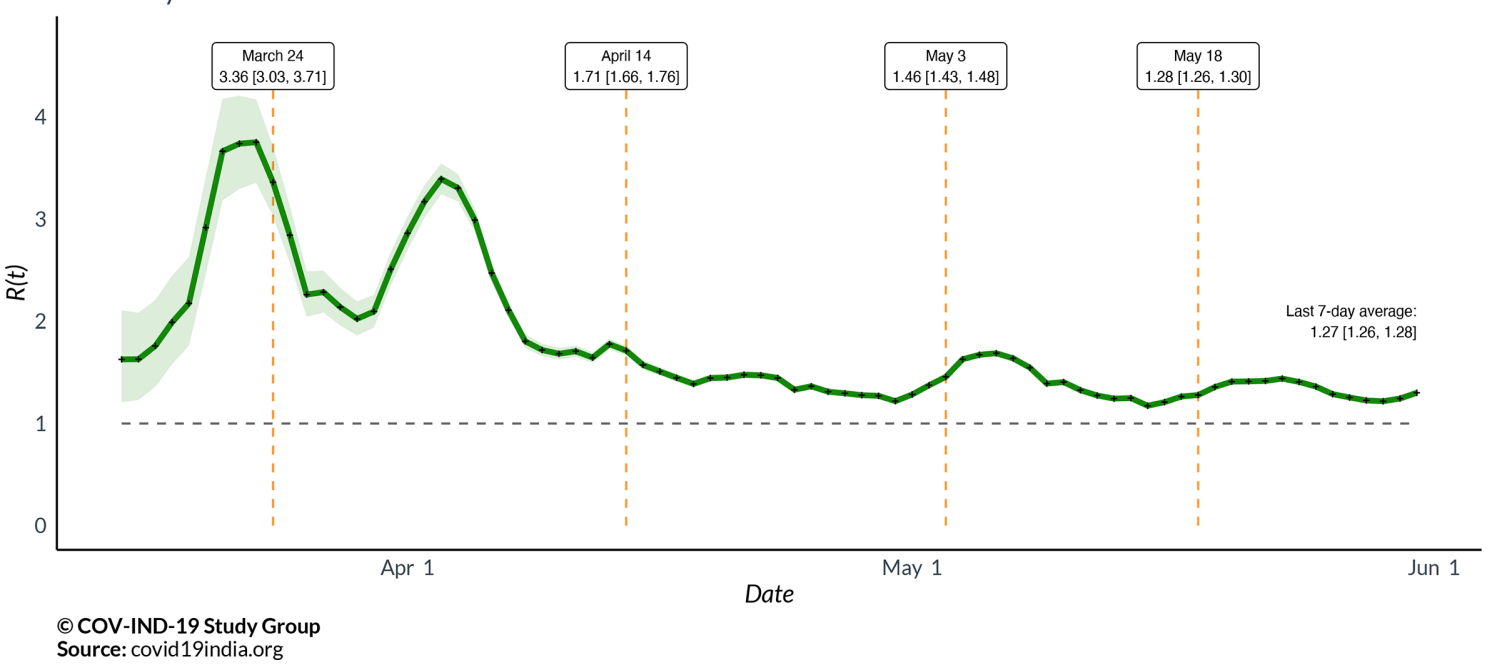

Figure 3 National estimates of doubling times and time-varying R. (A) Estimated doubling times of total number of COVID-19 cases in India, with averages for the prelockdown and postlockdown periods and past 7-day average as of 31 May. (B) Estimated time-varying $\mathrm{R}$ (effective basic reproduction number) for COVID-19 in India with averages for the prelockdown and postlockdown periods and past 7 -day average as of 31 May, along with $95 \%$ Cls.

window. This measure gives the number of days it would take for total cases to double if its trajectory remained as observed in the past week, and an increase in the DT is evidence of the pandemic slowing down. We use a descriptive measure as well as fit a log-linear model to estimate the DT (see online supplemental material). We also use a Bayesian sequential method to estimate the time-varying effective basic reproduction number, $\mathrm{R}$, which measures the average number of persons infected by an infected individual. When $\mathrm{R}$ falls below 1 , the epidemic starts slowing down. ${ }^{21}$ The estimation of the time-varying $\mathrm{R}$ is performed using the EpiEstim package in $\mathrm{R}$ and daily case count data from COVID-19 India. ${ }^{521}$ In particular, we used the vectors of daily new cases as our input using the 'parametric_SI' estimation method and a 5-day window ('estimate_R' function, which was used to describe the progression of the outbreak in Wuhan).$^{22}$ We also use a gamma distribution prior with a mean of 7 days and an SD of 4.5 days, based on research by Wu and colleagues, for the generation time (a distribution of the onset of disease used to estimate R). ${ }^{23}$

\section{Testing summaries}

In order to understand the testing landscape, we compute the proportion of population tested, test positivity rates (TPRs) and quantify testing metrics (number of tests, TPR, percentage of population tested) at the national and state levels. We also introduce a metric of testing shortfall which can be used after lockdown during the state of control of a pandemic to ensure sustained control of the TPR at a target level (eg, in May WHO recommended this target to be set at $\left.5 \%{ }^{24}\right)$. This may be useful for India after the daily incidence curve turns the corner which unfortunately did not happen during our study period of lockdown. 
A Doubling time
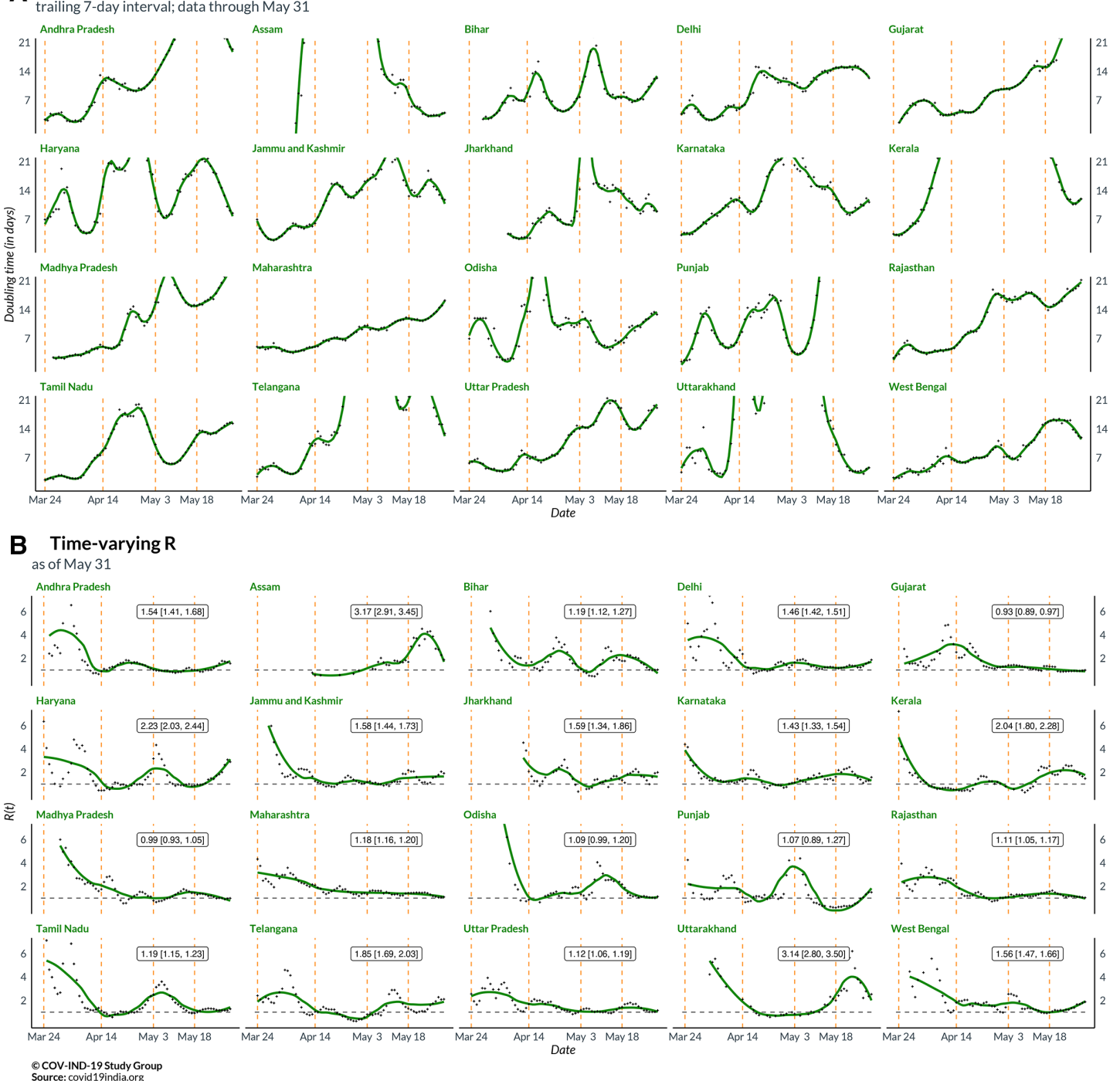

Figure 4 State-wise estimates of doubling times and time-varying R. (A) Estimated doubling times of total number of COVID-19 cases in 20 Indian states and union territories. (B) Estimated time-varying R (effective basic reproduction number) for COVID-19 in 20 Indian states and union territories along with 95\% Cls.

The detailed definition of each reported metric and methods for computing corresponding measures of uncertainty are presented in the online supplemental methods. All our analyses use data available from 15 March until 31 March, 31 March being the day India's strict national lockdowns ended and 'unlocking' started.

\section{Patient and public involvement}

No patient involvement.

\section{RESULTS}

\section{Total number of cases and deaths}

India had reported its first case of COVID-19 on 30 January. The first death from COVID-19 was reported on 12 March. In the second week of May, India recorded the highest growth in case counts among Asian countries. ${ }^{19}$ As of 9 June, only four countries (USA, Brazil, Russia and UK) had recorded more cases than India. ${ }^{25}$ Online supplemental figure 1 presents national trends of the COVID-19 outbreak in India by plotting the cumulative number of confirmed cases, fatalities and recovered cases. To highlight the pronounced geographic pattern across states not visible in online supplemental figure 1, figure 1 compares the daily profile of the pandemic at the national level with four states: two that are doing well (Kerala and Punjab) and two that have been hit hard (Maharashtra and Delhi) in terms of the same three counts. It is clear that Punjab has been doing well and has experienced the first initial peak, Kerala seems to have many new cases after the strong initial control, Maharashtra has an increasing trend that seems to be stabilising while Delhi has a high number of cases with a sudden jump in case counts near the end of the nationwide lockdown. Since Maharashtra contributes nearly $35 \%-40 \%$ of India's total number of cases, the national pattern has more resemblance to Maharashtra. Two crucial points emerge from the geographic pattern. First, the concentration of the case load 
among the top 10 states has remained relatively stable, at around $90 \%$ of the national case count, over this 2-month period. Second, the membership of the top 10 states has changed gradually—even as Maharashtra, Delhi and Uttar Pradesh have continued to figure in the list at all four lockdown markers. Online supplemental figures 2 and 3 plot cumulative case and death counts, respectively, across states and over time to highlight these geographic patterns.

\section{Case fatality rates}

Figure 2A (CFR1) and online supplemental figure 4 (CFR2) present forest plots of the two estimates of the CFR, along with $95 \%$ CIs, for the 20 states/union territories and for the nation as a whole. Using CFR1, there are several states with CFR1 above 3\%: Gujarat (6.2\%), West Bengal (5.8\%), Madhya Pradesh $(4.3 \%)$ Maharashtra $(3.4 \%)$ and Telangana $(3.0 \%)$. Similarly, the same five states plus Delhi have elevated CFR2 estimates (above 6\%).

\section{DT and reproduction number}

Figure 3A plots the estimated DTs and figure 3B plots the estimated time-varying $\mathrm{R}$ nationally. Since reliable estimates of DT and R require many days of data, figure 3A,B starts on 15 March. In both, we report the estimate (along with the 95\% CIs for R) on 24 March, 14 April, 3 May and 18 May corresponding to the initial lockdown and subsequent extensions, in order.

The time series patterns of estimated DT and R nationally show that the lockdown did slow down the spread of the pandemic. It took about 2 weeks for the DT to start moving up in a sustained manner. Since early April, the DT has increased from about 5 to over 14 days by the end of May (figure 3A). Turning to figure 3B, we see that the estimated value of $\mathrm{R}$ fell over the first lockdown from 3.36 (95\% CI 3.03 to 3.71 ) on 24 March to 1.71 (95\% CI 1.66 to 1.76) on 14 April, with substantial fluctuation in between. Since then, the estimated $\mathrm{R}$ has fallen at a slower pace. The trailing 7-day average value of $\mathrm{R}$ for the week ending on 31 May is 1.27 (95\% CI 1.26 to 1.28 ).

These national patterns hide substantial state-level variations, observable in state level (figure 4A (DT) and figure 4B (R)). Figure 4A shows that estimated DTs have mostly increased, with Assam, Delhi, Haryana, Odisha and Uttarakhand being some noteworthy exceptions. Figure $4 \mathrm{~B}$ indicates that starting from higher values, estimates of $\mathrm{R}$ have generally fallen across all states. Again, there are significant differences across states-some states continue to have high values (eg, Maharashtra), and some others, after a period of low estimates of $\mathrm{R}$, have reverted to relatively high value (eg, Kerala).

Figure 2B,C presents forest plots of the average value of the DT (with 7-day range) and estimated R (with 95\% CI) over the week before 31 May, respectively. Only two states have estimated R below 1 (Madhya Pradesh and Gujarat) and four states have estimated R over 2 (Assam, Uttarakhand, Haryana and Kerala).

\section{Testing coverage and TPR}

Going by national-level data, India seems to be doing fairly well in terms of TPRs. Since mid-April, India's TPR has fluctuated around 0.04 (figure 5). This is lower than many European and North American countries at that time, and significantly lower than its neighbours, like Bangladesh and Pakistan. ${ }^{8}{ }^{26}$ But this national trend hides the wide variation across states. Online supplemental figure 5 plots the TPR over time for our sample of 20 states/ union territories, exhibiting obvious and striking statewide variations (with recent estimates being summarised in figure 2D). Rising TPR is noted in most of these states where the pandemic is geographically concentrated. Important examples are Delhi, Gujarat, Maharashtra and Tamil Nadu, which have both high case counts and high/rising TPRs. Bihar, Telangana and Uttarakhand, with relatively low case counts so far, are witnessing rising TPRs, as seen in table 2, which also contains the proportion of population tested by 31 May across each state (online supplemental table 1 is an updated version of this table with data through 15 September to have the latest assessment).

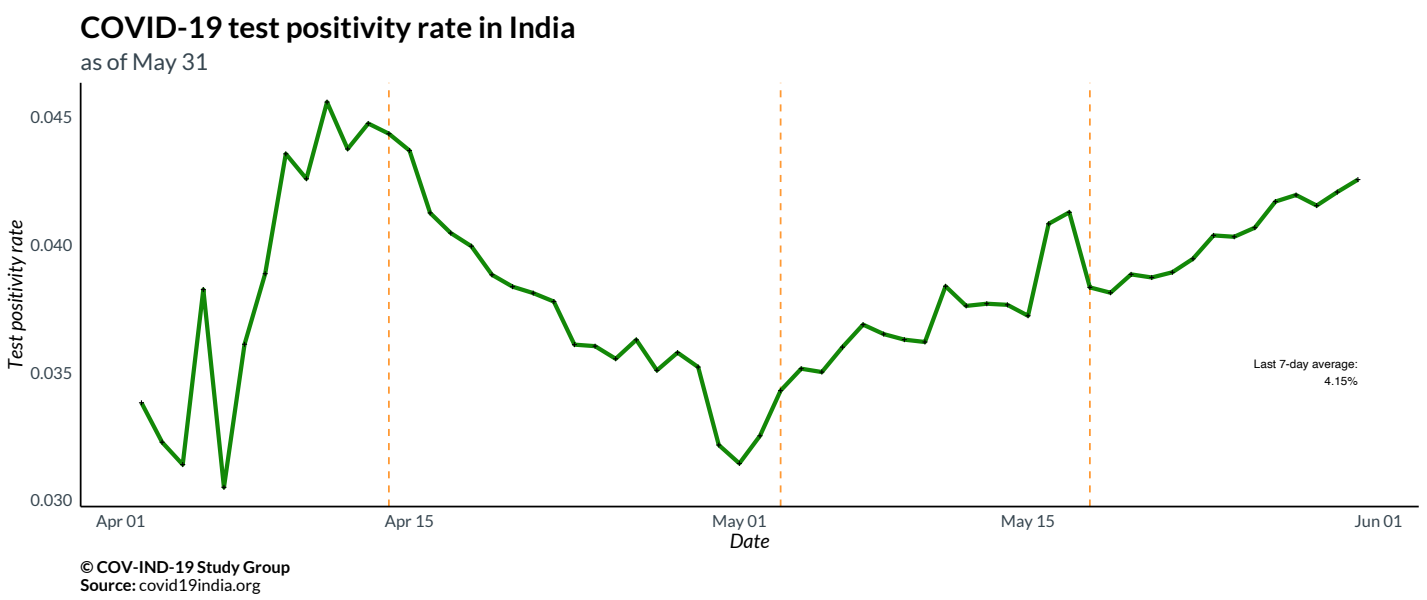

Figure 5 Time series plot of test positivity rates for India over the period between 1 April and 31 May. 
Table 2 COVID-19 metrics table for India and the 20 states with the most cumulative case counts as of 31 May 2020

\begin{tabular}{llllllll}
\hline & \multicolumn{2}{l}{ Metrics } & & & & \\
Location & $\mathbf{R}$ & Doubling time (days) & CFR & Test positivity rate & Total tested & Population & PPT (\%) \\
\hline National estimate & 1.27 & 14.4 & 0.028 & 0.042 & 3737027 & 1332830000 & 0.28 \\
Maharashtra & 1.18 & 13.8 & 0.034 & 0.136 & 463177 & 122153000 & 0.38 \\
\hline Delhi & 1.46 & 14.2 & 0.024 & 0.086 & 212784 & 19814000 & 1.07 \\
Gujarat & 0.93 & 25.6 & 0.062 & 0.079 & 211930 & 67936000 & 0.31 \\
\hline Tamil Nadu & 1.19 & 14.6 & 0.008 & 0.044 & 491962 & 75695000 & 0.65 \\
\hline Madhya Pradesh & 0.99 & 21.5 & 0.043 & 0.049 & 167808 & 82232000 & 0.2 \\
\hline Bihar & 1.19 & 9.9 & 0.006 & 0.044 & 75737 & 119520000 & 0.06 \\
\hline Uttar Pradesh & 1.12 & 17.4 & 0.027 & 0.028 & 289892 & 224979000 & 0.13 \\
\hline West Bengal & 1.56 & 14.2 & 0.058 & 0.026 & 203751 & 96906000 & 0.21 \\
\hline Rajasthan & 1.11 & 19.1 & 0.022 & 0.022 & 409777 & 77264000 & 0.53 \\
\hline Punjab & 1.07 & 73.5 & 0.020 & 0.029 & 87852 & 29859000 & 0.29 \\
Uttarakhand & 3.14 & 3.9 & 0.006 & 0.021 & 30438 & 11141000 & 0.27 \\
\hline Andhra Pradesh & 1.54 & 23.3 & 0.017 & 0.008 & 372748 & 52221000 & 0.71 \\
\hline Assam & 3.18 & 3.5 & 0.002 & 0.009 & 109097 & 34293000 & 0.32 \\
\hline Haryana & 2.23 & 12.8 & 0.010 & 0.015 & 118138 & 28672000 & 0.41 \\
\hline Jharkhand & 1.59 & 10.1 & 0.008 & 0.008 & 65886 & 37403000 & 0.18 \\
\hline Jammu and Kashmir & 1.58 & 14.5 & 0.011 & 0.013 & 171045 & 13203000 & 1.3 \\
\hline Karnataka & 1.43 & 10.4 & 0.016 & 0.010 & 293575 & 65798000 & 0.45 \\
\hline Kerala & 2.04 & 11.8 & 0.008 & 0.015 & 77508 & 35125000 & 0.22 \\
\hline Odisha & 1.09 & 12.0 & 0.005 & 0.012 & 152131 & 43671000 & 0.35 \\
\hline Telangana & 1.85 & 18.2 & 0.030 & NA & NA & 37220000 & NA \\
\hline
\end{tabular}

CFR, case fatality rate; NA, not applicable; PPT, proportion of population tested.

\section{Summary state-level dashboard: comprehensive display of metrics}

With a complete data tsunami, different metrics telling us different features of the pandemic and a rapidly evolving landscape, we offer a summary dashboard (figure 2) for the states and the nation according to various metrics. This captures a snapshot of where things stand across states and the nation, with daily updates available in our app hosted at covind19.org. ${ }^{19}$

Figure 2A shows CFR1 along with the 95\% CI. While the all-India CFR1 on 31 May was 2.84\%, state-level CFR1s ranged from $6.2 \%$ (Gujarat) to $0.2 \%$ (Assam). Figure 2B shows the 7-day average DT along with the range. The quickest DT is in Assam (3.5 days, range: 3.1, 4.0) while the slowest DT is in Punjab (73.5 days, range: 51.6, 97.5). The national estimate is 14.4 days (range: 13.2, 15.2), with about half of states having DT exceeding 14 days.

Figure 2C shows the 7-day average $\mathrm{R}$ along with the 95\% CIs. We see that 7-day average estimates range from 0.93 (95\% CI 0.89 to 0.97 ) in Gujarat to 3.17 (95\% CI 2.91 to 3.45) in Assam, with a national estimate of 1.27 (95\% CI 1.26 to 1.28 ). Figure 2B,C exhibits how the DT, a function of cumulative cases, is less sensitive to daily movements than R. For example, Kerala has done well controlling the outbreak in terms of DT, but a small recent increase in observed cases results in a 7-day average $\mathrm{R}$ estimate close to 2.

Figure 2D shows the 7-day average TPR along with the range. The lowest 7-day average TPR is seen in Andhra Pradesh $(0.83 \%$, range: $0.81 \%, 0.85 \%)$, with the highest being seen in Maharashtra (13.63\%, range: $13.25 \%$, $14.07 \%)$. Generally, states with larger cumulative case counts are seen to have higher TPRs. A high TPR most likely indicates inadequate levels of testing relative to the size of the outbreak. Thus, states with large number of reported cases are also likely to be suffering from low testing relative to the size of the outbreak in these states. The national 7 -day average TPR is $4.15 \%$ (range: $4.03 \%, 4.26 \%)$.

It is important to consider these metrics together, keeping their nuances in mind:

- CFR1 is an indicator of the fatality associated with the epidemic, but its value is sensitive to the number of tests being performed. A high CFR1 might very well arise from inadequate testing. Hence, the CFR1 is best used in conjunction with some measure of adequate testing.

- $\mathrm{R}$ can indicate a recent outbreak but is sensitive to the level of daily cases being observed (ie, a state/union territory with few cases can have a high R). In parallel, 
DT is a longer term measure since it is a function of cumulative cases (ie, this metric is more robust to fluctuations in recent daily cases). These are relative metrics and do not inform us about projected healthcare needs.

- TPR is both a function of the size of the outbreak in an area and the number of tests being performed. A higher TPR can indicate insufficient levels of testing and selective testing of symptomatic patients but also a good predictor of an emerging outbreak when large numbers of tests are done.

\section{DISCUSSION}

While it is common for analysts and policymakers to predict a peak for the COVID-19 in India, ${ }^{27} 28$ our analysis shows that the concept of a peak for the whole country is, at best, ambiguous. Differences in estimates of $\mathrm{R}$ (figure 4B) and estimated DTs (figure 4A) suggest that peaks will vary across states. Predictions from the extended SIR (eSIR) ${ }^{89}$ model available at covind19.org show that peak in case counts might start as early as the end of July in some states and go all the way to October in many others. (For a description of the method and the parameter settings used for the prediction models, please refer to the online supplemental methods and accompanying online supplemental figures 6 and 7.) Some states like Punjab have already experienced their first peak. These predictions are in line with basic intuition about the dynamics of the pandemic in India. Initial cases were imported, and the initial growth was limited to a few states which saw the arrival of international travellers. These initial cases seeded the epidemic and saw the explosion of cases. With the non-medical intervention of lockdown, mobility was limited at the macro level (interstate, intercity), which reduced transmission rates (figures 3 and 4). Prelockdown infections and micromobility resulted in growth of cases within states; notably, the top 10 states on 18 April and 31 May are largely the same. Now that we are in the targeted lockdown phase, internal migration will start playing an increasingly important role in the spread of the pandemic.

India has a large migrant worker population. Estimates of out-of-state and out-of-district migrants ranged from 60 to 80 million in 2011, and average work-related migration flows between states over the period 2011-2016 were about 9 million per year. ${ }^{30}$ With the easing of lockdown and work slowly resuming, a large migrant population will soon start travelling back to their workplaces and India could see the next surge in cases in states home to higher numbers of migrant workers. At the time of writing this paper in June this was our prediction. Indeed, at the time of revising the paper, we created pie charts in online supplemental figure 8 which show the shift in contributions of cases by state over time (through 15 September) which support our previous conjecture. Additional references on COVID-19 models incorporating migration and mobility can be found in online supplemental table $2{ }^{31-35}$ A combined and rigorous strategy of testing suspected patients, tracing contacts of patients and isolating infected persons can effectively break the chain of transmission and slow down the pandemic. Intensifying government messages on social distancing, mask wearing, avoiding large indoor gatherings and hygiene can allow the country to reopen safely. In a country like India, which can ill afford the severe economic disruption caused by a lockdown, this alternative approach has much to recommend itself. ${ }^{36}$

Regarding testing, the most common approach is to track the TPR, that is, fraction of positives in the total number of persons tested. ${ }^{37}$ High and/or rising TPRs indicate that either community prevalence is truly rising or that the level of testing is inadequate relative to the size of the outbreak so only symptomatic cases are being tested. Steady decline in TPR to 5\% or less for at least 14 consecutive days may indicate the pandemic is in a control phase, ${ }^{24}$ that is, an indication that effective $\mathrm{R}$ is declining and less than 1 . The testing shortfall metric can then be gainfully employed to determine the number of tests that need to be done randomly in the community for surveillance during a control phase. In order to devise a testing strategy, it is important not just to think about the number of tests but consider various types of tests, including rapid antigen test, RT-PCR test and cost-efficient testing strategies such as pooled testing, stratified periodic sampling to capture asymptomatic individuals. The goal of testing be it for clinical diagnostic purpose, screening or surveillance should be clear. The testing shortfall metric may indicate that we need to carry out a large number of tests that we do not have resources for, but this number can inform us when and where to scale up syndromic surveillance using community and government healthcare workers.

The estimated prevalence of the disease by TPR will usually be an overestimate of the 'true' prevalence rate due to testing of the symptomatic individuals who are more likely to have an active infection. On the other hand, under-reporting of silent or covert infections and lack of testing of mildly symptomatic individuals is a major challenge in estimating the true prevalence and infection fatality rate (IFR). The extent of under-reporting in terms of the number of confirmed cases and deaths is a pertinent metric in this context, and the under-reporting factor possibly varies across the states. Although we did not attempt to estimate the under-reporting factor in this paper, largely because our predictions come from an eSIR model that does not naturally model the asymptomatic infections and hence does not provide estimates of the true unreported number of cases, there exist modifications of such model-based approaches providing an estimate of the covert infections. An SEIR model applied to data from Wuhan, China, has earlier provided estimates of the under-reporting factor in terms of cases and deaths. ${ }^{38}$ In one of our recent works, we extended this SEIR model to account for misclassifications due to imperfect diagnostic testing and computed revised estimates of the under-reporting factor for cases and deaths using data from Delhi, the national capital region of India. ${ }^{39}$ 
We also performed validation for these model-based estimates using estimated seroprevalence information from a serosurvey performed in the region. ${ }^{40}$ Another recent study has used a multicountry-modified SEIR model to estimate COVID-19 under-reporting across 86 countries and has reported significant variability between the countries in terms of the estimated under-reporting factor. ${ }^{41}$ The effect of under-reporting of cases and deaths on IFRs can be found in online supplemental table 3. Depending on the degree of under-reporting for cases and deaths, the IFR ranges from $0.1 \%$ to $1.0 \%$. One has to remember that even with a low IFR of around $0.1 \%$, if $50 \%$ of people in India get infected, the nation will incur 670000 deaths.

Given the spatial and temporal pattern of the pandemic's spread, it is extremely important to prioritise policies. Resources must be mobilised to help one cluster of states and then move to the next cluster. It might be useful for the central government and the Indian Council of Medical Research to classify states in terms of the phases of the epidemic. Even as the worst-hit states are being addressed, the next set could be put on high alert. It is this dynamic policy intervention that will be required to deal effectively with the cascading pattern of the pandemic across Indian states (refer to table 2 to see state-level variation).

In implementing such a dynamic policy, it is extremely important to facilitate replication of successful strategies across states. Kerala's rapid response in terms of testing, contact tracing and quarantining; Odisha and Kerala's use of local governance structures and community health networks for surveillance and dissemination of correct information; Punjab's use of data analytics and districtlevel granular contact tracing, tracking and isolation-all these experiences will be of use in other states that are likely to see a surge in cases in the coming weeks.

There are several strengths of this work. First, it provides a comprehensive nationwide as well as state-level evaluation of the effect of India's national lockdown (Ghosh et $a l^{10}$ is another notable state-level analysis but focuses on forecasting rather than retrospective evaluation) on COVID-19 outbreak in India using an ensemble of metrics. These metrics can aid policymakers to track and assess the spread of the outbreak and identify areas where interventions may play an important mitigating role. Second, these metrics are publicly available and displayed via interactive visualisation tools that are daily updated at covind19.org ${ }^{19}$ to help inform dynamic policymaking and intervention towards containment and mitigation. Third, our state-level analysis highlights heterogeneity of outbreak progression across India and the concept of a cascade of 'peaks' across states instead of a solitary and unique national peak for the daily virus incidence curve.

There are also some limitations to this work. First, the metrics presented do not include predictions of future daily active cases and thus do not inform us about projected healthcare needs. We also refrain from predicting fatalities. The nature of this paper is more of retrospective evaluation than prospective forecasting. Second, our methods do not account for age-sex structure and mobility patterns in India. A full spatiotemporal model with more granular data is in order. Third, the quality of the data is in question with the existence of evidence that reported case counts are significantly lower than true case counts. However, this data set is the most comprehensive and regularly updated data set on COVID-19 in India and the undercount is likely missing asymptomatic cases rather than symptomatic cases. Attempting to correct for misclassification and other data errors is beyond the scope of this paper. Finally, we consider a narrow evaluation of the lockdown in terms of COVID-19-related outcomes using data up to 31 May. There are many long-term and broader consequences of the lockdown that this paper fails to capture.

The success of some states gives us hope that there are strategies to beat this insidious virus that have worked in a low-resource setting. Resources can be mobilised and optimally deployed to address the acute situations in high-density population areas like Maharashtra, Gujarat and Delhi. In all these efforts, nuanced statelevel summaries offer their utility to inform national policies.

\section{Author affiliations}

${ }^{1}$ Department of Biostatistics, University of Michigan, Ann Arbor, Michigan, USA

${ }^{2}$ Center for Precision Health Data Science, University of Michigan, Ann Arbor, Michigan, USA

${ }^{3}$ Department of Economics, University of Massachusetts Amherst, Amherst, Massachusetts, USA

${ }^{4}$ Department of Epidemiology, Johns Hopkins University, Baltimore, Maryland, USA

${ }^{5}$ Department of Biostatistics, Johns Hopkins University, Baltimore, Maryland, USA

Twitter Maxwell Salvatore @MaxSalTweets, Debashree Ray @DrDebashreeRay, Soumik Purkayastha @penguinhologram, Rupam Bhattacharyya @rupam_mmb and Bhramar Mukherjee @BhramarBioStat

Contributors DB and BM conceptualised this study. MS, SP, MK and RB curated the data. MS, RB and MK analysed the data. BM participated in funding acquisition, investigation and provided resources. DB, BM, MS and RB developed the methodology. MS, DR and BM administered the project. MS and MK created the software/app. DB, DR and BM supervised the study. DR, MS, RB, MK and BM helped in the visualisation. DB, MS, RB, SP, DR and BM wrote the original draft. All authors reviewed and edited the final manuscript.

Funding This study was funded by University of Michigan Precision Health Initiative, Michigan Institute of Data Science, National Cancer Institute (P30 CA 046592), University of Michigan Institute for Healthcare Policy and Innovation, University of Michigan School of Public Health and University of Michigan Rogel Cancer Center.

Competing interests None declared.

Patient consent for publication Not required.

Provenance and peer review Not commissioned; externally peer reviewed.

Data availability statement Data are available in a public, open access repository. We use publicly available data for all our analyses. All source codes and interactive plots are available at covind19.org. Nationwide and state-wide COVID-19 case data: https://www.covid19india.org/Interactive. Plots and dashboard: http://covind19.org/. Source codes: https://github.com/umich-cphds/cov-ind-19.

Supplemental material This content has been supplied by the author(s). It has not been vetted by BMJ Publishing Group Limited (BMJ) and may not have been peer-reviewed. Any opinions or recommendations discussed are solely those of the author(s) and are not endorsed by BMJ. BMJ disclaims all liability and responsibility arising from any reliance placed on the content. Where the content includes any translated material, BMJ does not warrant the accuracy and reliability of the translations (including but not limited to local regulations, clinical guidelines, 
terminology, drug names and drug dosages), and is not responsible for any error and/or omissions arising from translation and adaptation or otherwise.

Open access This is an open access article distributed in accordance with the Creative Commons Attribution Non Commercial (CC BY-NC 4.0) license, which permits others to distribute, remix, adapt, build upon this work non-commercially, and license their derivative works on different terms, provided the original work is properly cited, appropriate credit is given, any changes made indicated, and the use is non-commercial. See: http://creativecommons.org/licenses/by-nc/4.0/.

\section{ORCID iDs}

Soumik Purkayastha http://orcid.org/0000-0002-3619-2804

Bhramar Mukherjee http://orcid.org/0000-0003-0118-4561

\section{REFERENCES}

1 Mayo Foundation for Medical Education and Research. Coronavirus disease 2019 (COVID- 19) - Symptoms and causes. Mayo Clinic, 2020. Available: https://www.mayoclinic.org/diseases-conditions/ coronavirus/symptoms-causes/syc-20479963

2 Hui DS, I Azhar E, Madani TA, et al. The continuing 2019-nCoV epidemic threat of novel coronaviruses to global health - The latest 2019 novel coronavirus outbreak in Wuhan, China. Int $\mathrm{J}$ Infect Dis 2020;91:264-6.

3 Chauhan N. After Covid-19 lockdown, plan to unlock India in phases, 2020.

4 Government of India, Ministry of Home Affairs. D.O. No. 40-3/2020-DM-I(A); 2020.

5 COVID-19 India. COVID-19 Tracker updates for India for State-wise and District-wise data, 2020. Available: https://www.covid19india. org/

6 Indian Council of Medical Research. Advisory on strategy for COVID-19 testing in India, 2020. Available: https://www.icmr.gov.in/ pdf/covid/strategy/Testing_Strategy_v6_04092020.pdf

7 Jewell NP, Lewnard JA, Jewell BL. Predictive mathematical models of the COVID-19 pandemic: underlying principles and value of projections. JAMA 2020;323:1893.

8 Ray D, Salvatore M, Bhattacharyya R, et al. Predictions, role of interventions and effects of a historic national lockdown in India's response to the COVID-19 pandemic: data science call to arms. Harv Data Sci Rev 2020;2020. doi:10.1162/99608f92.60e08ed5. [Epub ahead of print: 09 Jun 2020].

9 Mitra A, Pakhare AP, Roy A, et al. Impact of COVID-19 epidemic curtailment strategies in selected Indian states: an analysis by reproduction number and doubling time with incidence modelling. Medrxiv. doi:10.1101/2020.05.10.20094946

10 Ghosh P, Ghosh R, Chakraborty B. COVID-19 in India: Statewise analysis and prediction. JMIR Public Health Surveill 2020;6:e20341.

11 Jakhar M, Ahluwalia PK, Kumar A. COVID-19 epidemic forecast in different states of India using Sir model. Medrxiv.

12 Gupta M. Transmission dynamics of the COVID-19 epidemic in India and modelling optimal lockdown exit strategies. Medrxiv.

13 Lathika Rajendrakumar A. Epidemic landscape and forecasting of SARS-CoV-2 in India. Medrxiv.

14 Government of India. Press information bureau VG/SNC/VM, 2020. Available: https://www.mha.gov.in/sites/default/files/PR NationalLockdown 26032020.pdf

15 Government of India,, Ministry of Home Affairs. D.O. No. 40-3/2020-DM-I(A), 2020. Available: https://www.mha.gov.in/sites/ default/files/MHA\%20DO\%20letter\%20dt.14.4.2020\%20to\% 20 Chief\%20Secretaries\%20and\%20Administrators $\% 20$ for $\%$ 20strict\%20implementation\%20of\%20Lockdown\%20Order\% 20during\%20extended\%20period.pdf

16 Government of India,, Ministry of Home Affairs. D.O. No. 40-3/2020-DM-I(A), 2020. Available: https://www.mha.gov.in/ sites/default/files/MHA\%200rder\%20Dt.\%201.5.2020\%20to\%
20extend\%20Lockdown\%20period\%20for\%202\%20weeks\%20w.e. f.\%204.5.2020\%20with\%20new\%20guidelines.pdf

17 Government of India,, Ministry of Home Affairs. D.O. No. 40-3/2020-DM-I(A), 2020. Available: https://www.mha.gov.in/sites/ default/files/MHAOrderextension_1752020_0.pdf

18 Roser M, Ritchie H, Ortiz-Ospina E, et al. Coronavirus Pandemic (COVID-19). Our World in Data, 2020. Available: https:// ourworldindata.org/coronavirus

19 COV-IND-19 Study Group. COVID-19 outbreak in India, 2020. Available: https://umich-biostatistics.shinyapps.io/covid19/

20 Ghani AC, Donnelly CA, Cox DR, et al. Methods for estimating the case fatality ratio for a novel, emerging infectious disease. Am J Epidemiol 2005;162:479-86.

21 Cori A, Ferguson NM, Fraser C, et al. A new framework and software to estimate time-varying reproduction numbers during epidemics. Am J Epidemiol 2013;178:1505-12.

22 Pan A, Liu L, Wang C, et al. Association of public health interventions with the epidemiology of the COVID-19 outbreak in Wuhan, China. JAMA 2020;323:1915.

$23 \mathrm{Wu}$ JT, Leung K, Bushman M, et al. Estimating clinical severity of COVID-19 from the transmission dynamics in Wuhan, China. Nat Med 2020;26:506-10.

24 Johns Hopkins University. Which U.S. states meet who recommended testing criteria? Coronavrus resource center, 2020. Available: https://coronavirus.jhu.edu/testing/testing-positivity

25 Johns Hopkins University. COVID-19 Dashboard by the center for systems science and engineering (CSSE) at Johns Hopkins University (JHU), 2020. Available: https://coronavirus.jhu.edu/map. $\mathrm{html}$

26 COV-IND-19 Study Group. Unlocking the 40-day national lockdown in India: there is no magic key. Medium 2020.

27 Belfin R. V, Brodka P, Radhakrishnan B, et al. COVID-19 peak estimation and effect of nationwide lockdown in India. Medrxiv 2020.

28 Paul A, Chatterjee S, Bairagi N. Prediction on Covid-19 epidemic for different countries: focusing on South Asia under various precautionary measures. Medrxiv 2020.

29 Wang Let al. An epidemiological forecast model and software assessing interventions on COVID-19 epidemic in China. Medrxiv 2020.

30 Government of India. Chapter 12. in economic survey 2016-17. Ministry of Finance, Government of India, 2017.

31 Maji A, Choudhari T, Sushma MB. Implication of repatriating migrant workers on COVID-19 spread and transportation requirements. Transportation Research Interdisciplinary Perspectives 2020;7:100187.

32 Kumar A. Modeling geographical spread of COVID-19 in India using network-based approach. Medrxiv 2020.

33 Pujari BS, Shekatkar SM. Multi-city modeling of epidemics using spatial networks: application to 2019-nCov (COVID-19) coronavirus in India. Medrxiv 2020

34 Gupta Set al. An India-specific compartmental model for Covid-19: projections and intervention strategies by incorporating geographical, Infrastructural and response heterogeneity. arXiv 2020.

35 Gupta AK, Sharma N, Verma AK. Spatial network based model forecasting transmission and control of COVID-19. Medrxiv 2020.

36 Cash R, Patel V. Has COVID-19 subverted global health? Lancet 2020;395:1687-8.

37 Stein R, Wroth C, Hurt AUS. Coronavirus Testing Still Falls Short. How's Your State Doing? 2020.

38 Hao X, Cheng S, Wu D, et al. Reconstruction of the full transmission dynamics of COVID-19 in Wuhan. Nature 2020;584:420-4.

39 Bhattacharyya R, Bhaduri R, Kundu R, et al. Reconciling epidemiological models with misclassified case-counts for SARSCoV-2 with seroprevalence surveys: a case study in Delhi, India. Medrxiv 2020.

40 Saxena A. Explained: Here are the key takeaways from Delhi's serological survey. The Indian Express, 2020.

41 Rahmandad H, Lim TY, Sterman J. Estimating COVID-19 underreporting across 86 nations: implications for projections and control. SSRN 2020. 